\title{
Third Order Markov Statistical Model and Stereoregularity Distribution of Poly(vinyl chloride)
}

\author{
Kunio Hisatani and Kunihiko OKaJima \\ Fundamental Research Laboratory of Natural and Synthetic Polymers, \\ Asahi Chemical Industry Co., Ltd., 11-7 Hacchownawate, Takatsuki, Osaka 569, Japan
}

(Received December 4, 1996)

\begin{abstract}
KEY WORDS Stereoregularity Distribution Analysis / Tacticity / Poly(vinyl chloride) / Third Order Markov Statistics /
\end{abstract}

Poly(vinyl chloride) (PVC) is still now one of the polymers used most widely over the world because of its excellent cost/performance despite of the arousing suspicion on its possible environmental hazard. As one important polymer characterization the stereoregularity distribution of PVC has been studied with NMR, ${ }^{1-3}$ and its septad stereoregularity distribution was carried out based on the NMR peak assignment using 1,4-dioxane$d_{8}$ as solvent by Elgert et al. by using ${ }^{13} \mathrm{C}$ NMR (125 $\mathrm{MHz}-\left\{{ }^{1} \mathrm{H}\right\}{ }^{13} \mathrm{C}$ ) in $1981 .{ }^{4}$

The stereoregularity distribution theory of polymers was first dealt with by Frisch et al. ${ }^{5}$ as early as 1950 s and the theory has been established in view of the mesoracemo concept (mr-type) and the D-L form consideration (DL-type) by many authors. ${ }^{6-10}$ The statistical models up to second order Markov statistics were comprehensively described by Frisch et al. ${ }^{11}$ Shelden et $a l .{ }^{12}$ and Shelden ${ }^{13}$ for the $m r$ - and DL-types, respectively. The expression of the stereoregularity distribution based on third order Markov statistics was tried to derive by Ito and Yamashita, ${ }^{14}$ but they only gave a comment that it was so highly complicated that it could be hardly expressed as an available mathematical description. A third order Markov statistics, however, might give a better basis for stereoregularity distribution to elucidate polymerization mechanism.

In the present article, a careful and strict mathematical derivation was made to describe the $m r$-type stereoregularity distribution of practical polymer in general based on a third order Markov statistics and its applicability was examined by using literature ${ }^{13} \mathrm{C}$ NMR data for PVC provided by Elgert et al. ${ }^{4}$

In Markov statistics for any polymer sequences, a model should be expressed in a way that each fraction of the given sequential arrangement with some definite chain length is written as a function of appropriate probability parameters. ${ }^{7}$ In the present third order Markov statistics for the $m r$-type conformational distribution, the probability parameters must satisfy the following equations:

$$
\begin{aligned}
& P_{(m / m m m)}+P_{(r / m m m)}=1 \\
& P_{(m / m m r)}+P_{(r / m m r)}=1 \\
& P_{(m / m r m)}+P_{(r / m r m)}=1 \\
& P_{(m / m r r)}+P_{(r / m r r)}=1
\end{aligned}
$$

$$
\begin{aligned}
& P_{(m / r m m)}+P_{(r / r m m)}=1 \\
& P_{(m / r m r)}+P_{(r / r m r)}=1 \\
& P_{(m / r r m)}+P_{(r / r r m)}=1 \\
& P_{(m / r r r)}+P_{(r / r r)}=1
\end{aligned}
$$

The number of independent parameters are eight. If eq $1 \mathrm{a}-\mathrm{h}$ are written as $a+\bar{a}=1, b+\bar{b}=1, c+\bar{c}=1$, $d+\bar{d}=1, e+\bar{e}=1, f+\bar{f}=1, g+\bar{g}=1$ and $h+\bar{h}=1$, respectively, then the tetrads must satisfy the following:

$$
\begin{aligned}
& {[\mathrm{mmm}]=[\mathrm{mmm}] a+[\mathrm{rmm}] e} \\
& {[\mathrm{mmr}]=[\mathrm{mmm}] \bar{a}+[\mathrm{rmm}] \bar{e}} \\
& {[\mathrm{mrm}]=[\mathrm{mmr}] b+[\mathrm{rmr}] f} \\
& {[\mathrm{mrr}]=[\mathrm{mmr}] \bar{b}+[\mathrm{rmr}] \bar{f}} \\
& {[\mathrm{rmm}]=[\mathrm{mrm}] c+[\mathrm{rrm}] g} \\
& {[\mathrm{rmr}]=[\mathrm{mrm}] \bar{c}+[\mathrm{rrm}] \bar{g}} \\
& {[\mathrm{rrm}]=[\mathrm{mrr}] d+[\mathrm{rrr}] \mathrm{h}} \\
& {[\mathrm{rrr}]=[\mathrm{mrr}] \bar{d}+[\mathrm{rrr}] \bar{h}}
\end{aligned}
$$

Combinations of any seven equations above and the following normalized condition,

$$
\begin{aligned}
& {[\mathrm{mmm}]+[\mathrm{mmr}]+[\mathrm{mrm}]+[\mathrm{mrr}]+[\mathrm{rmm}]} \\
& \quad+[\mathrm{rmr}]+[\mathrm{rrm}]+[\mathrm{rrr}]=1
\end{aligned}
$$

will lead to expressions for tetrads:

$$
\begin{aligned}
{[m m m] } & =(c f+\bar{f} g) e / \bar{a} D_{3}^{m r} \\
& =\{g+f(c-g)\} e / \bar{a} D_{3}^{m r}=z_{g} e / \bar{a} D_{3}^{m r} \\
{[m m r] } & =[r m m]=(c f+\bar{f} g) / D_{3}^{m r} \\
& =\{g+f(c-g)\} / D_{3}^{m r} \quad=z_{g} / D_{3}^{m r} \\
{[m r m] } & =(b g+f \bar{g}) / D_{3}^{m r} \quad \\
& =\{f+g(b-f)\} / D_{3}^{m r} \quad=z_{f} / D_{3}^{m r} \\
{[m r r] } & =[r r m]=(1-b c-\bar{c} f) / D_{3}^{m r} \\
& =\{\bar{f}+c(f-b)\} / D_{3}^{m r} \quad=z_{\bar{f}} / D_{3}^{m r} \\
{[r m r] } & =(1-b c-\bar{b} g) / D_{3}^{m r} \quad \\
& =\{\bar{g}+b(g-c)\} / D_{3}^{m r} \quad=z_{\bar{g}} / D_{3}^{m r} \\
{[r r r] } & =(1-b c-\bar{c} f) \bar{d} / h D_{3}^{m r} \\
& =\{\bar{f}+c(f-b)\} \bar{d} / h D_{3}^{m r}=z_{\bar{f}} \bar{d} / h D_{3}^{m r}
\end{aligned}
$$


Table I. ${ }^{13} \mathrm{C}$ NMR chemical shift in the region of $\mathrm{CH}$ carbon of septad fraction of poly(vinyl chloride) evaluated by Elgert $e t \mathrm{al}^{4}$

\begin{tabular}{|c|c|c|c|c|}
\hline \multirow{2}{*}{$\begin{array}{c}\text { Signal } \\
\text { No. }\end{array}$} & \multirow{2}{*}{$\begin{array}{l}\text { Chemical } \\
\text { shift/ppm }\end{array}$} & \multirow{2}{*}{ Pentad or septad sequence } & \multicolumn{2}{|c|}{ Mol fraction of sequence } \\
\hline & & & Cited & Normalized \\
\hline 1 & 58.20 & rmrrmr & 0.023 & $0.022_{8}$ \\
\hline 2 & 58.11 & rmrrmm & 0.034 & $0.033_{7}^{\circ}$ \\
\hline 3 & 58.02 & $m m r r m m+r r m m+r r r m r$ & 0.103 & $0.102_{1}$ \\
\hline 4 & 57.94 & $m r r r m m+m r r r m r$ & 0.074 & $0.073_{3}$ \\
\hline 5 & 57.83 & $r r r r$ & 0.091 & $0.090_{2}$ \\
\hline 6 & 57.34 & $m m m r m m+m m m r m r$ & 0.037 & $0.036_{7}$ \\
\hline 7 & 57.25 & $r m m r m m+r m m r m r+m r m r m m+m r m r m r$ & 0.114 & $0.113_{0}$ \\
\hline 8 & 57.17 & rrmrmr + rrmrmm & 0.073 & $0.072_{3}$ \\
\hline 9 & 57.10 & $m m r r$ & 0.087 & $0.086_{2}$ \\
\hline 10 & 57.00 & $r m r r$ & 0.204 & $0.202_{2}$ \\
\hline 11 & 56.49 & rmmmmr & 0.011 & $0.010_{9}$ \\
\hline 12 & 56.43 & $m m m m m r$ & 0.015 & $0.014_{9}$ \\
\hline 13 & 56.37 & mmmmmm & 0.004 & $0.004_{0}$ \\
\hline 14 & 56.26 & $r m m m r+r m m m r m$ & 0.050 & $0.049_{6}$ \\
\hline 15 & 56.20 & $m m m m r r+m m m m r m$ & 0.035 & $0.034_{7}$ \\
\hline 16 & 56.04 & $r m m r$ & 0.054 & $0.053_{5}$ \\
\hline
\end{tabular}

where

$$
\begin{aligned}
D_{3}^{m r}= & 2\{1+(c-g)(f-b)\}+\{g+f(c-g)\}(1+e / \bar{a}) \\
& +\{\bar{f}+c(f-b)\}(1+\bar{d} / h)
\end{aligned}
$$

$z_{\bar{d}}=1+\bar{d} / h, z_{e}=1+e / \bar{a}, z_{f}=f+g(b-f), z_{\bar{f}}=\bar{f}+c(f-b)$, $z_{g}=g+f(c-g), z_{\bar{g}}=\bar{g}+b(g-c)$ are used. These expressions are quite simple. If $z_{3}=1+(c-g)(f-b)$ are introduced, the following relations hold among them:

$$
\begin{aligned}
b z_{g}+f z_{\bar{g}} & =z_{f} \\
\bar{b} z_{g}+\bar{f} z_{\bar{g}} & =z_{\bar{f}} \\
c z_{f}+g z_{\bar{f}} & =z_{g} \\
\bar{c} z_{f}+\bar{g} z_{\bar{f}} & =z_{\bar{g}} \\
z_{f}+z_{\bar{f}} & =z_{g}+z_{\bar{g}}=z_{3} \\
D_{3}^{m r} \quad & =2 z_{3}+z_{e} z_{g}+z_{\bar{d}} z_{\bar{f}}
\end{aligned}
$$

An alternation of $m$ to $r$ (or $r$ to $m$ ) corresponds to exchange between $a, b, c, d, e, f, g, h$, and $\bar{h}, \bar{g}, \bar{f}, \bar{e}, \bar{d}, \bar{c}$, $\bar{b}, \bar{a}$, respectively, and the parameters $z_{e}, z_{f}$, and $z_{g}$ should be simultaneously exchanged with $z_{\bar{d}}, z_{\bar{g}}$, and $z_{\bar{f}}$, respectively. All the pentads are:

$$
\begin{aligned}
& {[\mathrm{mmmm}]=z_{g} a e / \bar{a} D_{3}^{m r}} \\
& {[\mathrm{mmmr}]=z_{g} e / D_{3}^{m r}} \\
& {[\mathrm{mmrm}]=z_{g} b / D_{3}^{m r}} \\
& {[\mathrm{mmrr}]=z_{g} \bar{b} / D_{3}^{m r}} \\
& {[\mathrm{mrmm}]=z_{f} c / D_{3}^{m r}} \\
& {[\mathrm{mrmr}]=z_{f} \bar{c} / D_{3}^{m r}} \\
& {[\mathrm{mrrm}]=z_{\bar{f}} d / D_{3}^{m r}} \\
& {[\mathrm{mrr}]=z_{\bar{f}} \bar{d} / D_{3}^{m r}} \\
& {[r m m m]=z_{g} e / D_{3}^{m r}} \\
& {[r m m r]=z_{g} \bar{e} / D_{3}^{m r}} \\
& {[r m r m]=z_{\bar{g}} f / D_{3}^{m r}} \\
& {[r m r r]=z_{\bar{g}} \bar{f} / D_{3}^{m r}}
\end{aligned}
$$

Table II. Values of $\delta_{\min }^{\prime}{ }^{\mathrm{a}} \times 1000$ for various $m r$-type Markov statistics of poly(vinyl chloride) sample

\begin{tabular}{ll}
\hline \multicolumn{1}{c}{ Statistics } & $\delta_{\min }^{\prime} \times 10^{3}$ \\
\cline { 2 - 2 } & $m r$-Type \\
\hline Bernoulli & $3.6_{1}$ \\
First order Markov & $2.1_{5}$ \\
Second order Markov & $1.6_{3}$ \\
Third order Markov & $1.3_{1}^{\mathrm{b}}$ \\
\hline
\end{tabular}

a $\delta_{\text {min }}^{\prime}$ means square root of $\left(X_{i}-Y_{i}\right) / N$, where $X_{i}$ and $Y_{i}$ mean observed and calculated fractions, respectively, and $N$, the number of fractions. ${ }^{\mathrm{b}}$ Underline, the most suitable value.

$$
\begin{aligned}
{[r r m m] } & =z_{\bar{f}} g / D_{3}^{m r} \\
{[r r m r] } & =z_{\bar{f}} \bar{g} / D_{3}^{m r} \\
{[r r r m] } & =z_{\bar{f}} \bar{d} / D_{3}^{m r} \\
{[r r r r] } & =z_{\bar{f}} \bar{d} \bar{h} / h D_{3}^{m r}
\end{aligned}
$$

The septads can be readily expressed in a similar manner. Selection of the most suitable statistics can be made in accordance with the method described in previous literature. ${ }^{15,16}$

Table I lists the ${ }^{13} \mathrm{C}$ NMR peak assignment of methine carbon based on septads and their molar fractions reported by Elgert et al., ${ }^{4}$ as well as normalized. Elgert et al. assigned these peaks by comparison of experimental and calculated peak intensities based on a Bernoulli distribution of stereoregularity. By assuming peak assignment is correct, statistical sequences were re-examined based on Bernoulli, first, second, and third order Markov statistics using their data and the resultant deviation $\delta_{\min }^{\prime}$ values are summarized in Table II. In general, the larger the number of political parameters, the more suitable the model becomes. However, if there are more sufficient data than the parameters, there exists certain meaning for the models in question while the values of the criterion function become significantly smaller.

It turns out that the third order Markov statistics is most probable, suggesting that at least a three-monomer- 
Table III. Comparison of chemical shifts of septads

\begin{tabular}{lcccc}
\hline & \multicolumn{3}{c}{ Relation between septad } \\
\cline { 2 - 5 } Sequence & $\Delta\left({ }^{*} m m m\right)-\Delta\left({ }^{*} m m r\right)^{\mathrm{a}}$ & $\Delta\left({ }^{*} m r m\right)-\Delta\left({ }^{*} m r r\right)$ & $\Delta\left({ }^{*} r m m\right)-\Delta\left({ }^{*} r m r\right)$ & $\Delta\left({ }^{*} r r m\right)-\Delta\left({ }^{*} r r r\right)$ \\
$m m r r m$ & - & - & $<$ & - \\
$m r r r$ & - & - & $=0$ & $<0$ \\
$r r r r$ & - & - & - & $=0$ \\
$m m r m$ & $>0$ & -0 & -0 & - \\
$r m r m$ & - & - & - & $=0$ \\
$m m r r$ & $=0$ & -0 & - & -0 \\
$r m r r$ & - & - & - & - \\
$m m m m$ & $<0$ & $=0$ & - & -
\end{tabular}

${ }^{\text {a } e . g ., ~} \Delta\left({ }^{*} m m m\right)-\Delta\left({ }^{*} m m r\right)$ represents $\Delta\left(? ?{ }^{*} m m m\right)-\Delta\left(? ?{ }^{*} m m r\right)$ where ? means either $m$ or $r$ to examine the influence of septad sequence end conformation. $>0$ means that an NMR peak due to septad sequence end of $m$ appears in the lower magnetic field than that of $r$.

unit-arrangement controls the reaction of next monomer at propagation end probably due to the electro-magnetical circumstances induced by the sequential arrangement. This further tells us that the NMR peak assignment expected from simple $m$ and $r$ connection concept is not always realized especially for longer sequences. For example, a close inspection of the peak assignment made by Elgert et al. reveals that among the septads belonging to $\mathrm{mmrm}$ pentad, $\mathrm{mmmrmm}$ and $m m m r m r$ are assigned to lower magnetic field peaks than rmmrmm and rmmrmr, respectively, and contrarily mmmmmm and mmmmmr belonging to $m m m m$ pentad are assigned to higher magnetic field peaks than $m m m m m r$ and rmmmmr, respectively. That is, if we mark the observation carbon nucleus by ${ }^{*},{ }^{*} m m m$ is assigned sometimes at higher field than $* m m r$ and another times at lower field than $*_{m m r}$. This relation is compiled in Table III. In all four kinds of combinations of *?? $m / * ? ? r$ (? means $m$ or $r$ ) there are some exceptions for relative chemical shift positions expected from simple $m$ and $r$ connection concept. However, even if we amend the peak assignment so as to match the above simple concept, we could not find any assignment which gives lower $\delta_{\min }^{\prime}$ than that obtained by Elgert et al.'s assignment. All the above facts and discussion lead us to the conclusion that their assignments are correct, although the methods to assign were too simple and primitive.

As mentioned before, since the stereoregularity distribution of PVC can be regarded to obey third order Markov statistics, the arrangements of three monomer units at the both sides of a monomer in question might influence the chemical shifts of the methine carbon in question.

The chemical shifts for peaks assigned by Elgert et al. are delicately influenced by whether septad stereoregularity end is $m$ or $r$, and even if we examine the septads with a conformation ( $m$ or $r$ ) end the chemical shifts seem to be dependent on the precedent sequential arrangement in a different manner. The situation is shown in Table III. Here, the movement of chemical shifts difference of central monomer unit when one monomer unit is added to end of one side from a central monomer in base pentad in either $m$ or $r$. Most conspicuous varieties are seen when ${ }^{*} r m$ is concerned in opposite side of sequential arrangement. This is quite similar tendency observed for pentad and then similar discussion on
Table IV. Optimized statistical parameters for various $m$ r-type Markov statistics of a poly(vinyl chloride) sample

\begin{tabular}{lll}
\hline \multicolumn{1}{c}{ Statistics } & \multicolumn{2}{c}{ Parameter } \\
\hline Bernoulli & $P_{m}$ & $0.42_{0}$ \\
\hline First order Markov & $P_{(m / m)}$ & $0.35_{3}$ \\
& $P_{(m / r)}$ & $0.44_{5}$ \\
\hline Second order Markov & $P_{(m / m m)}$ & $0.42_{2}$ \\
& $P_{(m / m r)}$ & $0.43_{6}$ \\
& $P_{(m / r m)}$ & $0.33_{6}$ \\
& $P_{(m / r r)}$ & $0.46_{1}$ \\
\hline & $P_{(m / m m m)}$ & $0.41_{1}$ \\
& $P_{(m / m m r)}$ & 0.673 \\
& $P_{(m / m r m)}$ & $0.35_{1}$ \\
& $P_{(m / m r r)}$ & $0.44_{5}$ \\
& $P_{(m / r m m)}$ & $0.40_{4}$ \\
& $P_{(m / r m r)}$ & $0.30_{7}$ \\
& $P_{(m / r r m)}$ & $0.39_{1}$ \\
& $P_{(m / r r r)}$ & $0.46_{3}$ \\
\hline
\end{tabular}

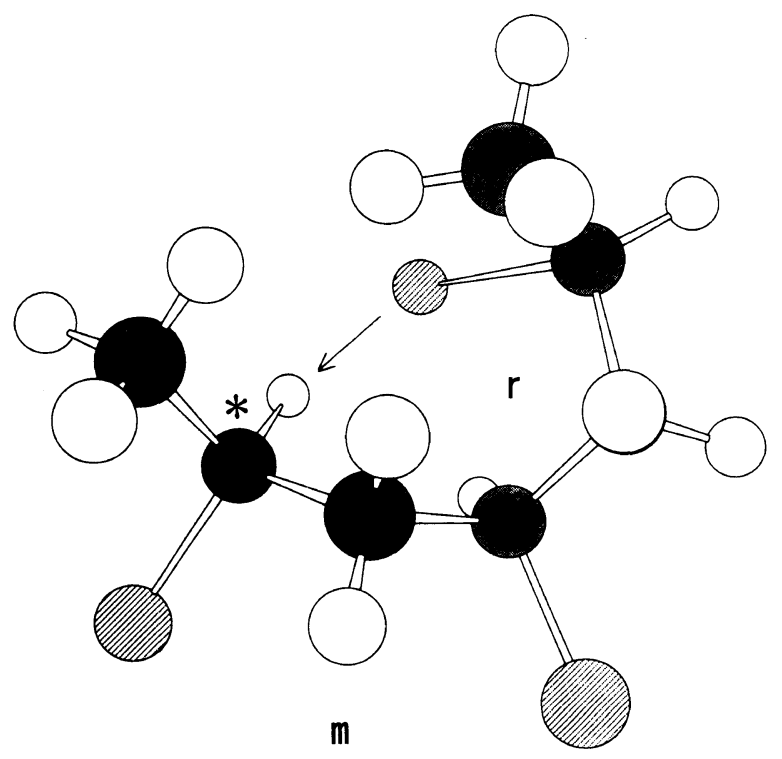

Figure 1. Interaction between hydrogen and chlorine alienated by consequent meso and racemo stereoregularity. ${ }^{*}$ means methine carbon.

septads is applicable in general. As a natural consequence, septads should also be discussed in view of molecular circumstances but it seems to be far beyond 
the present scope of this communication because of the above complicated circumstances.

The values of probability parameters obtained by third order Markov statistics are listed in Table IV, as well as the probability parameters obtained based on first and second order Markov statistics. The third order Markov statistics reveals that $P_{(m / m m r)}\left(0.67_{3}\right), P_{(r / m r m)}\left(0.64_{9}\right)$, $P_{(r / r m r)}\left(0.69_{3}\right)$, and $P_{(r / r r m)}\left(0.61_{0}\right)$ are relatively high. These parameters correspond to all probabilities of propagation triad ends characterized by $\mathrm{rm}$ and $\mathrm{mr}$, suggesting that such triad propagating ends tend to control the stereospecific polymerization of vinyl chloride with some propagating feature to form rather heterotactic structure. We suppose that such feature might relate to an idea that these triad end species can take some 7-membered ring (Figure 1), which is different probably from the triad ends having $\mathrm{mm}$ and $r r$. The tendency resembles to the stereospecific polymerization of acrylonitrile by coordination-anionic method reported by Ono et al. ${ }^{17}$ and Nakano, ${ }^{18}$ and that shown for methacrylonitrile reported by Joh et al. ${ }^{19}$ and Soum et al. ${ }^{20}$

\section{REFERENCES}

1. C. J. Carman, A. R. Tarpley, and J. H. Goldstein, Macromolecules,
4, 445 (1971)

2. I. Ando, Y. Kato, and A. Nishioka, Makromol. Chem., 177, 2759 (1976).

3. W. H. Starnes, F. C. Schilling, K. B. Abbas, R. E. Cais, and F. A. Bovey, Macromolecules, 12, 556 (1979).

4. K.-F. Elgert, R. Kosfeld, and W. E. Hull, Polym. Bull., 4, 281 (1981).

5. H. L. Frisch, C. Shuerch, and M. Szwarc, J. Polym. Sci., 11, 559 (1953).

6. B. D. Coleman, J. Polym. Sci., 31, 155 (1958).

7. R. L. Miller and L. E. Nielsen, J. Polym. Sci., 46, 303 (1960).

8. J. W. L. Fordham, J. Polym. Sci., 39, 321 (1959).

9. F. P. Price, J. Chem. Phys., 36, 209 (1962).

10. A. Miyake and R. Chujo, J. Polym. Sci., 46, 163 (1960).

11. H. L. Frish, C. L. Mallows, and F. A. Bovey, J. Chem. Phys., 45, 1565 (1966)

12. R. Shelden, J. Furukawa, and T. Fueno, J. Polym. Sci., A-2, 7, 763 (1969).

13. R. Shelden, J. Polym. Sci., A-2, 7, 1111 (1969).

14. K. Ito and Y. Yamashita, J. Polym. Sci., A, 3, 2165 (1965).

15. K. Kamide and K. Hisatani, Polym. J., 24, 1377 (1992).

16. K. Hisatani, K. Okajima, and K. Kamide, Polym. J., 27, 728 (1995).

17. H. Ono, K. Hisatani, and K. Kamide, Polym. J., to be prepared.

18. Y. Nakano, Doctoral Thesis to Kanazawa Univ. (1995).

19. Y. Joh, T. Yoshihara, Y. Kotake, F. Ide, and K. Nakatsuka, J. Polym. Sci., Polym. Lett. Ed., 4, 673 (1966).

20. A. Soum and M. Fontanille, Am. Chem. Soc., Symp. Ser., 166, 239 (1981). 